\title{
Clinical comparision of intravesical hyaluronic acid and hyaluronic acid-chondroitin sulphate therapy for patients with bladder pain syndrome/interstitital cystitis
}

\author{
Ömer Gülpınar, MD; Aytaç Kayış, MD; Evren Süer, MD; Mehmet Illker Gökçe, MD; Adil Güçal Güçlü, MD; \\ Nihat Arıkan, MD
}

Department of Urology, Ankara University, Ankara, Turkey

ite as: Can Urol Assoc J 2014;8(9-10):e610-4. http://dx.doi.org/10.5489/cuaj.2036

Published online September 9, 2014.

\section{Abstract}

Introduction: Patients with a history of bladder pain syndrome/ interstitial cystitis (BPS/IC) and who responded poorly or unsatisfactorily with previous treatment were compared taking intravesical hyaluronic acid (HA) or hyaluronic acid-chondroitin sulphate (HA-CS).

Methods: Patients were treated with intravesical instillation with $50 \mathrm{~mL}$ sterile sodium hyalurinic acid (Hyacyst, Syner-Med, Surrey, UK) $(\mathrm{n}=32)$ and sodium hyaluronate $1.6 \%$ sodium chondroitin sulphate $2 \%$ (laluril, Aspire Pharma, UK) $(\mathrm{n}=33)$. Intravesical instillations were performed weekly in first month, every 15 days in the second month and monthly in third and fourth months, for a total of 8 doses. Patients were evaluated using a visual analog pain scale (VAS), interstitial cystitis symptom index (ICSI), interstitial cystitis problem index (ICPI), voiding diary for frequency/ nocturia, cystometric bladder capacity and voided volume at the beginning and at 6 months. All patients had a potassium sensitivity test (PST) initially. Wilcoxon and Mann-Whitney $U$ tests were used for statistical analysis.

Results: In total, 53 patients met the study criteria. There were 30 patients in the HA-CS group (mean age: 48.47) and 23 patients in the HA group (mean age: 49.61$)(p>0.05)$. The initial PST was positive in $71.7 \%$ patients $(38 / 53)$ overall with no difference between groups ( $p>0.05)$. Responses for VAS, ICCS, ICPS, 24-hour frequency/nocturia statistically improved in both groups at 6 months. There was no significant difference in symptomatic improvement $(p>0.05)$. Eight patients had mild adverse events. Conclusion: HA and HA/CS instillation can be effective in BPS/IC patients who do not respond to conservative treatment. An important limitation of our study is that the HA dosage of the 2 treatment arms were different. It would be more appropriate with same HA dosage in both groups; however, there was no commercially available glycosaminoglycan (GAG) substance with same HA dosage for single and combination therapy. Large, long-term randomized studies are required to determine if there is a difference between these treatments.

\section{Introduction}

Bladder pain syndrome/interstitial cystitis (BPS/IC) is a debilitating, chronic disease characterized by urinary urgency, frequency, bladder and pelvic pain and has a negative impact on quality of life. ${ }^{1}$ Prevalence rates are $2.71 \%$ and $1.22 \%$ in women and men, respectively, although these rates depend on the definitions used. ${ }^{2}$ The etiology of BPS/IC is unknown, although there are several theories, including autoimmune response, mast cell activation, neuropathic changes, occult infection, toxic substances in the urine, and a primary defect in the glycosaminoglycan (GAG) layer of the bladder mucosa. ${ }^{3,4}$ Although the etiology is unclear, the role of the urinary epithelium in the BPS/IC is well-established. ${ }^{5-8}$ It has been hypothesized that a defective or abnormal GAG layer, a mucus layer important for the integrity of the bladder mucosa, plays a role by allowing urinary solutes, such as potassium, to diffuse into the subepithelial tissues inducing an inflammatory reaction and eventually stimulating a peripheral neuropathy. The major classes of GAG include hyaluronic acid $(\mathrm{HA})$, heparin sulphate, heparin, chondroitin 4-sulphate, chondroitin 6-sulphate, dermatan sulphate and keratan sulphate. $^{9}$

To improve the integrity and function of the bladder lining, intravesical GAG replacement therapies are one of the recommended treatment options for patients with BPS/IC refractory to conventional therapy. ${ }^{10}$ Currently, chondroitin sulphate (CS), heparin, $\mathrm{HA}$, and pentosanpolysulphate, and combinations of two GAGs (CS and $\mathrm{HA}$ ) are the available substances with different effectiveness rates in patients with BPS/IC.

As there are presently no studies comparing these substances in BPS/IC patients, physicians may have a difficult time choosing the optimal GAG replacement treatment for their patients. Therefore, it makes sense to evaluate these different products in clinical trials. In this study, we compared the clinical efficacy of HA and HA-CS, 2 commercially 
available intravesical agents, in BPS/IC patients who had poor response or were unsatisfied with previous treatment.

\section{Methods}

Patients with BPS/IC were included in the study. The clinical signs and symptoms of BPS/IC are "chronic pelvic pain, pressure, or discomfort perceived to be related to the urinary bladder accompanied by at least one other urinary symptom, such as persistent urge to void or frequency for at least 24 weeks," ${ }^{\prime 11}$ an average pain/discomfort score of $\geq 4$ on a 0 to 10 visual analogue scale (VAS, where 0 is no pain and 10 is maximum pain), voiding frequency $\geq 12$ times/24 hours, a negative urine pregnancy test, a sterile urine culture at least 30 days prior to treatment and an inadequate clinical response after 6 months of conservative treatment. The conservative treatment could have included 1 of the following: patient education, timed voiding and behavioural modification therapy, dietary restrictions, stress reduction and/or oral therapy with tricyclic antidepressants, antihistamines, antimuscaranic (anticholinergic) agents, pentosanpolysulphate, or analgesics. Patients were excluded if they were pregnant, breastfeeding, had a current urinary tract infection (UTI), a diagnosis of chemical cystitis, tuberculosis or radiation cystitis, or urolithiasis, urothelial cancer, sexually transmitted infection, endometriosis, urethral diverticulum, or bladder symptoms. Cystoscopy with hydrodistension and potassium sensitivity test (PST) was performed prior to treatment. The study was approved by the hospital ethics committee, and all patients gave written informed consent.

We investigated data from patients treated with either a sterile solution of $\mathrm{HA}(1.6 \% \mathrm{w} / \mathrm{v}, 800 \mathrm{mg} / 50 \mathrm{~mL})$ and CS $(2 \% \mathrm{w} / \mathrm{v}, 1 \mathrm{~g} / 50 \mathrm{~mL})$ (Ialuril, Aspire Pharma, UK) or a sterile solution of HA (120 mg HA in $50 \mathrm{~mL}$ ) (Hyacyst, Syner-Med, Surrey, UK) between March 2010 and March 2012. The patients were followed prospectively and the data were analyzed retrospectively. Solutions were administered via hydrophilic $12 \mathrm{Fr}$ Foley catheters and performed by a urology resident. Patients were instructed to retain the instillation volume for at least 60 minutes. Intravesical instillations were performed weekly in first month, every 15 days in the second month and monthly in third and fourth months, for a total of 8 doses. Patients were evaluated using a VAS, interstitial cystitis symptom index (ICSI), interstitial cystitis problem index (ICPI) 3-day voiding diary (24-hour frequency/nocturia, mean voided volume) and cystometric bladder capacity at the beginning and after 6 months of the therapy. Vital signs and any adverse events were recorded at each treatment session.

The primary outcome of the study was the change of pain VAS score from baseline compared to week 24. Secondary outcomes included comparison of daily urinary frequency/ nocturia, ICSI and ICPI scores, mean voided volume and cystometric bladder capacity from baseline to week 24 .

Sample size was estimated as 40 (20 patients in each group) with power of 0.80 and an effect size of $50 \%$ (change in VAS score from 8 to 4 points after the treatment is expected).

Baseline factors were compared with the t-test and the Mann-Whitney rank sum test, and differences between treatment outcomes were calculated with the Mann-Whitney rank sum test. Proportions of responders were calculated with the Fisher exact test; $p<0.05$ was considered significant. Statistical analysis was performed with SPSS, v.16.0 statistical software (SAS Institute, Cary, NC).

\section{Results}

Between March 2010 and March 2012, 65 patients received treatment with the intravesical HA or HA-CS. In the end, data from 53 female patients were analyzed, as 12 were lost to follow-up (Fig. 1). There were 30 patients in the HA-CS group with mean age of 48.47 and 23 patients in the HA group with mean age of $49.61(p>0.05)$. PST was positive for 23 patients $(76.6 \%)$ and 15 patients $(65.2 \%)$ in HA-CS and HA groups, respectively ( $p>0.05)$. Of the 53 patients, 38 patients $(71.7 \%)$ had positive PST. There was no statistically significant difference between the initial findings of the 2 groups $(p>0.05)$ (Table 1). Former treatments in HA-CS group were dietary restrictions and amitriptyline $(n=18)$, dietary restrictions and hydroxyzine hydrochloride $(n=8)$ and oral pentosanpolysulphate $(n=4)$. Dietary restrictions and analgesics $(n=6)$, dietary restrictions and amitriptyline $(n=8)$, dietary restrictions and hydroxyzine hydrochloride $(n=4)$ and oral pentosanpolysulphate $(n=5)$ were the former treatments of the HA group. We compared the responses at baseline and at 6 months (Table 2, Fig. 2). The primary outcome VAS of pain decreased significantly at 6 months in both groups ( 8.5 to $4[p=0.001]$ and 9 to $4[p=0.001]$ in the HA-CS and HA groups, respectively) (Fig. 2a). Secondary outcomes, including 24-hour frequency/ nocturia, ICSI and ICPS, decreased significantly in both treatment arms $(p<0.05)$. Cystometric bladder capacity and mean voided volume increased, yet these results were not statistically significant ( $p>0.05)$ (Fig. 2b). No statistically significant differences in primary and secondary outcomes were seen between groups $(p>0.05)$ (Table 2).

In all, 8 patients $(15 \%)$ reported 12 mild adverse events, including UTI $(\mathrm{n}=6)$ and bladder symptoms, such as increased frequency, urgency or incontinence $(n=6)$.

\section{Discussion}

GAG layer replenishment therapy is widely accepted as therapy for patients with BPS/IC who have poor or inadequate response to conventional therapy. ${ }^{10}$ There are 4 different commercially available products for GAG replenishment, 


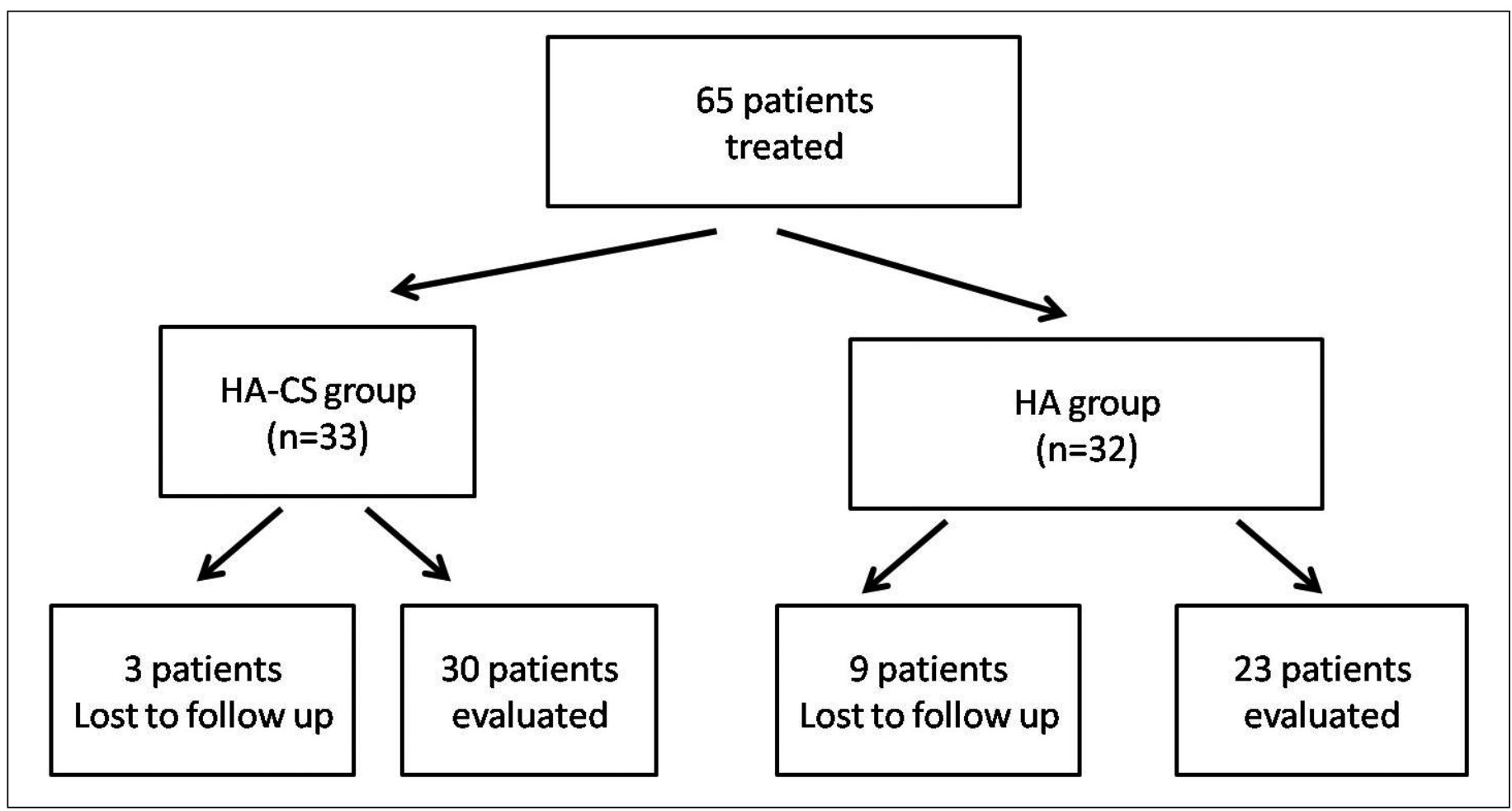

Fig. 1. STROBE (STrengthening the Reporting of OBservational studies in Epidemiology) diagram of eligible participants. HA: hyaluronic acid; CS: chondroitin sulphate.

including CS, heparin, HA and pentosanpolysulphate. Each product has different concentrations and dosage formulations. Recently, a combination of CS and HA is the latest commercially available product.

Intravesical HA was the first GAG substance used for BPS/ IC. The first study was published by Morales and colleagues in 1996; the authors found a complete or partial response rate of $71 \%$ for up to 1 year. ${ }^{12} \mathrm{HA}$ is widely studied and has shown a wide range of symptom improvement, from $30 \%$ to $85 \% .{ }^{3,13,14}$ Recently, Engelhardt and colleagues reported their long-term results of intravesical HA therapy and they observed a $50 \%$ complete bladder symptom remission at the 5-year follow-up without any additional therapy, while $41.7 \%$ with symptom recurrence improved with HA maintenance therapy. ${ }^{15}$ On the other hand, in a double-blind, placebo-controlled, multicentre clinical study of different hyaluronic acid preparations (40 or $200 \mathrm{mg} / \mathrm{cc}$ ), no significant efficacy of sodium hyaluronate compared to placebo was found for IC patients; however, further details, including patient selection, inclusion/exclusion criteria, definition of improvement/success, are not available. ${ }^{16}$

Intravesical CS therapy efficiency was evaluated by Steeinhoff and colleagues in an open-label 12-month study. In this study, 18 patients with $40-\mathrm{mL}$ instillations of CS $0.2 \%$ weekly for 4 weeks and then monthly for 12 months, we assessed. The authors found a response rate for symptom improvement of $67 \% .{ }^{17}$ In an uncontrolled open multicen- tre study of 53 IC patients, instillations of CS $2 \%$ produced a $60 \%$ response rate at 6 months. ${ }^{18}$ In contrast, a recently published randomized controlled trial (RCT) failed to show superiority of CS $2.0 \%$ over control after 6 weeks of treatment. ${ }^{19}$ In that study, most patients reported a clinical benefit, but the difference between treatment and control group was not statistically significant.

A combination of 2 GAG contains CS $(2.0 \%)$ and low molecular weight $\mathrm{HA}(1.6 \%)$ is the latest available substance

Table 1. Pre-treatment characteristics of the 2 groups

\begin{tabular}{lccc}
\hline Parameters & $\begin{array}{c}\text { HA-CS, } \mathbf{n = 3 0} \\
\mathbf{( 5 6 . 6 \% )}\end{array}$ & $\begin{array}{c}\text { HA, } \mathbf{n}=\mathbf{2 3} \\
\mathbf{( 4 3 . 3 \% )}\end{array}$ & $\begin{array}{c}\boldsymbol{p} \\
\text { value }\end{array}$ \\
\hline Age, years & $48.47 \pm 14.73$ & $49.61 \pm 16.59$ & 0.792 \\
VAS & $8.2 \pm 2.09$ & $8 \pm 2.88$ & 0.703 \\
Micturition frequency & $15.1 \pm 7.03$ & $14.8 \pm 6.47$ & 0.978 \\
(24-hour): mean \pm SD & $3.1 \pm 2.40$ & $3.1 \pm 2.55$ & 0.971 \\
Nocturia, mean \pm SD & $15.2 \pm 3.57$ & $15.2 \pm 3.95$ & 0.870 \\
ICSI & $13.2 \pm 2.52$ & $13.3 \pm 2.70$ & 0.827 \\
ICPI & $301.6 \pm 121.92$ & $286.2 \pm 127.24$ & 0.654 \\
Cystometric capacity & & & \\
(mL), mean \pm SD & $154.6 \pm 94.17$ & $151 \pm 90.59$ & 0.914 \\
Voided volume (mL), & & & \\
mean \pm SD & $76.7 \%$ & $65.2 \%$ & 0.082 \\
PST positivity rate (\%) &
\end{tabular}

HA: hyaluronic acid; CS: chondroitin sulphate; VAS: visual analog pain scale; ICSI: interstitial cystitis symptom index; ICPI: interstitial cystitis problem index; PST: potassium sensitivity test; SD: standard deviation; SD: standard deviation. 


\begin{tabular}{|c|c|c|c|c|c|c|c|}
\hline & \multicolumn{3}{|c|}{ HA-CS } & \multicolumn{3}{|c|}{ HA } & \multirow{2}{*}{$\begin{array}{c}\text { HA-CS vs HA } \\
p \text { value }\end{array}$} \\
\hline & Initial visit & 6 months & $p$ value & Initial visit & 6 months & $p$ value & \\
\hline VAS & $8.2 \pm 2.09$ & $4.9 \pm 2.08$ & 0.000 & $8 \pm 2.88$ & $4.5 \pm 2.52$ & 0.000 & 0.761 \\
\hline Frequency: mean \pm SD & $15.1 \pm 7.03$ & $12 \pm 4.51$ & 0.002 & $14.8 \pm 6.47$ & $11.8 \pm 4.52$ & 0.010 & 0.698 \\
\hline Nocturia: mean \pm SD & $3.1 \pm 2.40$ & $1.4 \pm 1.28$ & 0.001 & $3.1 \pm 2.55$ & $1.3 \pm 1.32$ & 0.002 & 0.861 \\
\hline ICSI & $15.2 \pm 3.57$ & $11.4 \pm 4.46$ & 0.001 & $15.2 \pm 3.95$ & $11.1 \pm 4.77$ & 0.003 & 0.891 \\
\hline ICPI & $13.2 \pm 2.52$ & $8.4 \pm 4.45$ & 0.000 & $13.3 \pm 2.70$ & $8.9 \pm 4.35$ & 0.000 & 0.665 \\
\hline Cystometric capacity $(\mathrm{mL})$, mean $\pm \mathrm{SD}$ & $301.6 \pm 121.92$ & $322.5 \pm 126.78$ & 0.130 & $\begin{array}{c}286.2 \pm \\
127.24\end{array}$ & $\begin{array}{c}322.4 \pm \\
119.12\end{array}$ & 0.135 & 0.973 \\
\hline Voided volume $(\mathrm{mL})$, mean $\pm \mathrm{SD}$ & $154.6 \pm 94.17$ & $173.7 \pm 86.10$ & 0.059 & $151 \pm 90.59$ & $\begin{array}{c}164.9 \\
\pm 80.22\end{array}$ & 0.555 & 0.473 \\
\hline
\end{tabular}

Results of the 2 different therapies compared in the last column. HA: hyaluronic acid; CS: chondroitin sulphate; VAS: visual analog pain scale; ICSI: interstitial cystitis symptom index; ICPI: interstitial cystitis problem index; PST: potassium sensitivity test; SD: standard deviation; SD: standard deviation.

for the GAG replenishment therapy. In an open-label single arm study by Porru and colleagues, the efficiency of intravesical CS/HA combination therapy was evaluated in 20 BPS/ IC patients. Parameters included VAS for pain and urgency, number of void per day, mean voiding volume, ICSI and Pain Urgency Frequency (PUF) Questionnaire; these scores showed statistically significant change after treatment compared with baseline. ${ }^{20}$ Cervigni and colleagues reported the long-term results of intravesical CS/HA therapy in 12 patients and they showed the sustained efficiency for 3 years in terms of mean number of voids per day and mean volume per void with the confirmation of quality of life assessments. ${ }^{21}$

Our study is the first study in the literature comparing the 2 different intravesical GAG substances to treat BPS/IC. We compared intravesical HA/CS combination and intravesical HA in patients with BPS/IC who had inadequate clinical response after 6 months of conservative treatment. Improvements regarding the VAS for pain, 24-hour fre-

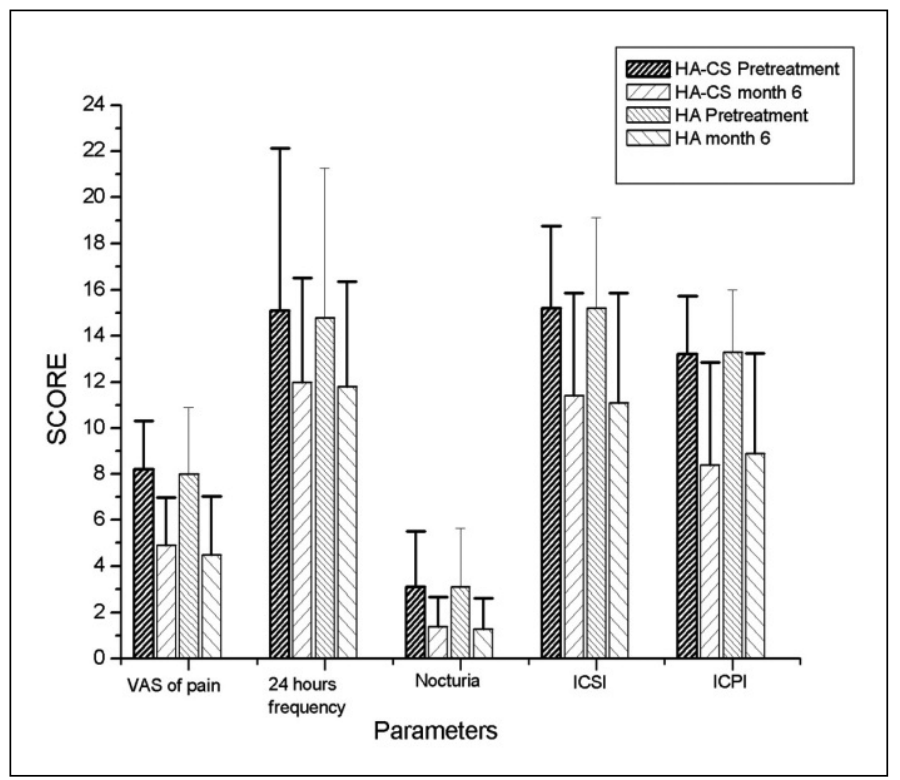

Fig. 2a. The primary outcome VAS of pain at 6 months. quency/nocturia, ICSI and ICPI were statistically significant at 6 months for both treatment arms. However, there was no difference between a CS/HA combination to $\mathrm{HA}$ in any parameter at the 6-month evaluation. Different from the HA, CS is the only sulphated GAG that is located on the bladder urothelial luminal surface and that contributed to urothelial barrier function..$^{22}$ Engels and colleagues demonstrated that CS treatment decreased the recruitment of inflammatory cells, including neutrophils and mast cells, to the suburothelial space in an acute cystitis model of the bladder. ${ }^{23}$ The loss of the impermeability barrier function of the urothelium ${ }^{8}$ and the inflammatory response of the bladder to the suburothelial urine leakage ${ }^{7}$ are well-established causes of BPS/ IC. Properties of CS combined with HA should maximize the efficiency of HA treatment of BPS/IC. However, we did not identify any short-term difference between $\mathrm{HA}$ alone and HA/CS. Further research with large prospective RCTs with long-term follow-up is required to provide a definitive answer.

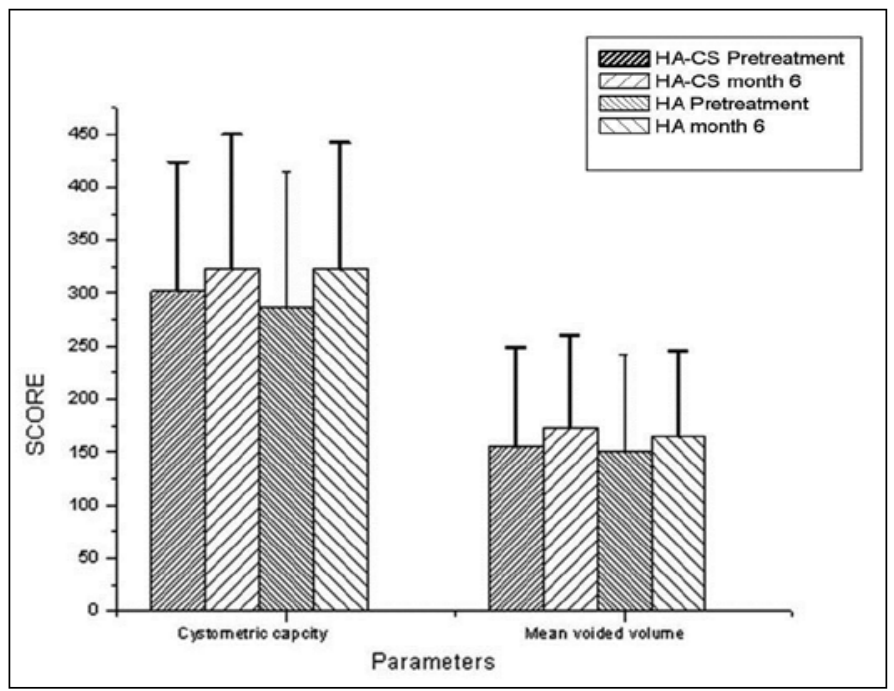

Fig. 2b. Cystometric bladder capacity and mean voided volume. 
An important limitation of our study is that the HA dosage of the 2 treatment arms were different $(120$ and $800 \mathrm{mg}$ in $\mathrm{HA}$ and $\mathrm{CS} / \mathrm{HA}$, respectively). It would be more appropriate with the same HA dosage in both groups; however, there was no commercially available GAG substances with the same HA dosage for single and combination therapy. Furthermore, our primary purpose was to see if there was any difference between the 2 available and reimbursable intravesical agents in our country. The duration of the follow-up period and the number of patients were limited in our study, but comparable to other series. ${ }^{18,19}$ The retrospective nature and lack of control group are other limitations; however, similar baseline group characteristics alleviate these handicaps. We also felt that a RCT design, where patients would be randomized to no treatment or placebo, may be difficult as these patients were in eevere pain and were desperate for treatment.

\section{Conclusion}

Intravesical HA and HA/CS therapy are effective treatment options for patients with BPS/IC who had inadequate response to conservative treatment, in the short term. Our study did not identify any differences. Further research with large prospective RCTs with long-term follow-up will be required to provide a definitive answer.

Competing interests: Dr. Gülpınar, Dr. Kayış, Dr. Süer, Dr. Gökçe, Dr. Güçlü and Dr. Arıkan all declare no competing financial or personal interests.

This paper has been peer-reviewed.

\section{References}

1. van de Merwe JP, Nordling J, Bouchelouche P, et al. Diagnostic criteria, classification, and nomenclature for painful bladder syndrome/interstitial cystitis: An ESSIC proposal. Eur Urol 2008;53:60-7. http:// dx.doi.org/10.1016/i.eururo.2007.09.019

2. Clemens JQ, Link CL, Eggers PW, et al. Prevalence of painful bladder symptoms and effect on quality of life in black, Hispanic and white men and women. J Urol 2007;177:1390-4. http://dx.doi. org/10.1016/i.juro.2006.11.084

3. Gupta SK, Pidcock L, Parr NJ. The potassium sensitivity test: A predictor of treatment response in intersititial cystitis. BJU Int 2005;96:1063-6. http://dx.doi.org/10.1111/i.1464-410X.2005.05776.x

4. Moldwin RM, Sant GR. Interstitial cystitis: A pathophysiology and treatment update. Clin Obstet Gynecol 2002;45:259-72. http://dx.doi.org/10.1097/00003081-200203000-00027
5. Slobodov G, Feloney $M$, Gran C, et al. Abnormal expression of molecular markers for bladder impermeability and differentiation in urothelium of interstitial cystitis patients. J Urol 2004;171:1554-8. http://dx.doi. org/10.1097/01.ju.0000118938.09119.05

6. Chai TC, Keay S. New theories in interstitial cystitis. Nat Clin Pract Urol 2004;1:85-9. http://dx.doi. org/10.1038/ncpuro0057

7. Hauser PJ, Dozmorov MG, Bane BL, et al. Abnormal expression of differentiation related proteins and proteoglycan core proteins in the urothelium of patients with intersititial cystitis. J Urol 2008; 179:764-9. http://dx.doi.org/10.1016/i.juro.2007.09.022

8. Parsons $\mathrm{CL}$. The role of a leaky epithelium and potassium in the generation of bladder symptoms in interstitial cystitis/overactive bladder, urethral syndrome, prostatitis and gynaecological chronic pelvic pain. BJU Int 2011;107:370-5. http://dx.doi.org/10.1111/i.1464-410X.2010.09843.x

9. Hurst RE. Structure, function and pathology of proteoglycans and glycosaminoglycans in the urinary tract. World J Urol 1994;12:3-10. http://dx.doi.org/10.1007/BF00182044

10. Fall M, Baranowski AP, Elneil S, et al. EAU guidelines on chronic pelvic pain. Eur Urol 2010;57:35-48. http://dx.doi.org/10.1016/j.eururo.2009.08.020

11. Hanno PM, Landis JR, Matthews-Cook Y, et al. The diagnosis of interstitial cystitis revisited: Lessons learned from the National Institutes of Health Interstitial Cystitis Database study. J Urol 1999;161:553-7. http:// dx.doi.org/10.1016/S0022-5347(01)61948-7

12. Morales A, Emerson L, Nickel JC, et al. Intravesical hyaluronic acid in the treatment of refractory intersitital cystitis. J Urol 1996;156:45-8. http://dx.doi.org/10.1016/S0022-5347(01)65933-0

13. Kallestrup EB, Jorgensen SS, Nordling J, et al. Treatment of interstitial cystitis with Cystistat: A hyaluronic acid product. Scand J Urol Nephrol 2005;39:143-7. http://dx.doi.org/10.1080/00365590410015876

14. Riedl CR, Engelhardt PF, Daha KL, et al. Hyaluronan treatment of interstitial cystitis/painful bladder syndrome. Int Urogynecol J Pelvic Floor Dysfunct 2008;19:717-21. http://dx.doi.org/10.1007/ s00192-007-0515-5

15. Engelhardt PF, Morakis N, Daha LK, et al. Long-term results of intravesicalhyaluronan therapy in bladder pain syndrome/interstitial cystitis. Int Urogynecol J 2011;22:401-5.

16. Hanno P, Baranowski A, Fall $M$, et al. Painful bladder syndrome (including interstitial cystitis). In: Abrams PH, Wein AJ, Cardozo L, editors: Incontinence. 3rd edition. Paris: Health Publications Limited; 2005:1456-520.

17. Steinhoff $G$, Ittah $B$, Rowan $S$. The efficacy of chondroitin sulphate $0.2 \%$ in treating interstitial cysititis. Can J Urol 2002;9:1454-8.

18. Nickel JC, Egerdie B, Downey J, et al. A real-life multicentre clinical practice study to evaluate the efficacy and safety of intravesical chondroitin sulphate for the treatment of interstitial cystitis. BJU Int 2009;103:56-60. http://dx.doi.org/10.1111/j.1464-410X.2008.08028.x

19. Nickel JC, Egerdie RB, Steinhoff $G$, et al. A multicenter, randomized, double-blind, parallel group pilot evaluation of the efficacy and safety of intravesical sodium chondroitin sulphate versus vehicle control in patients with interstitial cystitis/painful bladder syndrome. Urology 2010;76:804-9. http://dx.doi. org/10.1016/i.urology.2010.03.016

20. Porru D, Leva F, Parmigiani $A$, et al. Impact of intravesical hyaluronic acid and chondroitin sulphate on bladder pain syndrome/interstitial cystitis. Int Urogynecol J 2012;23:1193-9.

21. Cervigni $M$, Natale F, Nasta L, et al. Intravesical hyaluronic acid and chondroitin sulphate for bladder pain syndrome/interstitial cystitis: Long-term treatment results. Int Urogynecol J 2012;23:1187-92.

22. Janssen $\mathrm{DA}$, van Wijk XM, Jansen $\mathrm{KC}$, et al.The distribution and function of chondroitin sulphate and other sulphated glycosaminoglycans in the human bladder and their contribution to the protective bladder barrier. J Urol 2013;189:336-42. htrp://dx.doi.org/10.1016/i.juro.2012.09.022

23. Engles CD, Hauser PJ, Abdullah SN, et al. Intravesical chondroitin sulphate inhibits recruitment of inflammatory cells in an acute acid damage "leaky bladder" model of cystitis. Urology 2012;79:483.e13-7.

Correspondence: Dr. Adil Güçal Gülịu, Department of Urology, Ankara University, Ankara, Turkey; dr.adil.guclu@gmail.com 